\title{
Remodelado auricular derecho y niveles plasmáticos de Galectina-3 se relacionan con la capacidad funcional de pacientes con hipertensión arterial pulmonar
}

\author{
Luigi Gabrielli ${ }^{1}$, Hugo Verdejo ${ }^{1}$, María Paz Ocaranza ${ }^{1}$, Pablo Sepúlveda ${ }^{2}$, Fernando Baraona ${ }^{1}$, \\ Manuel Salinas ${ }^{1}$, Rodrigo Saavedra ${ }^{1}$, Silvana Llevaneras ${ }^{1}$, Clara Quiroga ${ }^{1}$, Bernardita Garayar ${ }^{1}$, \\ Sergio Lavandero ${ }^{3-4}$ y Pablo Castro ${ }^{1}$
}

\footnotetext{
1 Advanced Center for Chronic Diseases, División de Enfermedades Cardiovasculares, Facultad de Medicina. Pontificia Universidad Católica de Chile, Santiago, Chile.

2 Hospital San Juan de Dios. Universidad de Chile.

3 Advanced Center for Chronic Diseases, Facultad de Ciencias Químicas y Farmacéuticas \& Facultad de Medicina, Universidad de Chile, Santiago, Chile.

4 Cardiology División. Department of Internal Medicine, University of Texas Southwestern Medical Center, Dallas, Texas, USA.
}

Introducción: En pacientes con hipertensión arterial pulmonar (HAP) Galectina- 3, biomarcador de fibrosis miocárdica, se ha asociado a marcadores ecocardiográficos de remodelado ventricular derecho. La relación entre Galectina- 3, remodelado auricular derecho (AD) y capacidad funcional (CF) en pacientes con HAP no ha sido explorado. El objetivo fue medir niveles de Galectina-3 y su relación con CF y remodelado $\mathrm{AD}$ en pacientes con HAP.

Métodos: Estudio prospectivo observacional en que se incluyeron 14 pacientes con HAP. En todos los pacientes se midieron los niveles de Galectina-3, proBNP, se evaluó la CF mediante test de caminata 6 minutos (TC6M) y se evaluó remodelado AD. Se consideraron para el análisis dos grupos según la distancia caminada en TC6M (> $200 \mathrm{~m}$ vs. $\leq 200 \mathrm{~m}$ ).
Resultados: La edad promedio fue $43 \pm 10$ años, el $84 \%$ mujeres. Los niveles de Galectina-3 fueron 16,1 $\pm 7,4 \mathrm{ng} / \mathrm{mL}$ y el TC6M fue $371 \pm 142 \mathrm{mts}$. Los pacientes con $\mathrm{TC} 6 \mathrm{M} \leq 200 \mathrm{~m}$ presentaron mayores niveles de Galectina-3 (27,3 $\pm 4,6$ vs $13,7 \pm 3,8 ; \mathrm{p}=0,006)$ y mayor volumen $\mathrm{AD}(151 \pm 21$ vs $94 \pm 43$; $\mathrm{p}=0,04)$. Además, se observó una correlación inversa entre el área $\mathrm{AD}$ y $\mathrm{TC} 6 \mathrm{M}(-0,71 ; \mathrm{p}=0,03)$.

Conclusión: Niveles elevados de Galectina-3 y parámetros de remodelado adverso en $\mathrm{AD}$ se relacionan con una menor $\mathrm{CF}$ en pacientes con HAP. Estos hallazgos apuntan a una mejor caracterización de pacientes con HAP y eventualmente la búsqueda de nuevos objetivos terapéuticos.

Palabras clave: Remodelado cardíaco, aurícula derecha, galectina-3, hipertensión pulmonar.

Correspondencia:

Dr. Luigi Gabrielli

Igabriel@uc.cl

Dr. Pablo F. Castro

pcastro@med.puc.cl.

División de Enfermedades Cardiovasculares,

Facultad de Medicina. Pontificia Universidad

Católica de Chile, Marcoleta 367, Santiago. Chile 


\section{Right atrial remodeling and plasma leves of Galectin-3 are related to functional capacity in patiensts with Pulmonary Artery Hypertension}

Background: Galectin-3 is a biomarker of myocardial fibrosis and has been associated with echocardiographic markers of right ventricular remodeling in patients with pulmonary artery hypertension (PAH). The association among Galectin-3 level, right atrial (RA) remodeling and functional capacity (FC) has not been explored. The objective was to measure plasma Galectin-3 concentrations and its relation with RA remodeling and FC in PAH patients.

Methods: This is a prospective observational study and 14 PAH patients were included. Galectin-3 and proBNP levels were measured in all patients. FC was estimated by the 6-minute walk test (6MWT) and used to define 2 groups of subjects ( $\leq 200 \mathrm{~m}$ or $>200 \mathrm{~m}$ ). RA area and volume were measured by echocardiography from a 4 chamber view.

Results: The average age was $43 \pm 10$ years, $84 \%$ of patients were female. Galectin-3 levels were $16.1 \pm 7.4$ $\mathrm{ng} / \mathrm{mL}$ and 6MWT was $371 \pm 142 \mathrm{~m}$. We observed an inverse correlation between RA area and 6MWT $(-0.71 ; \mathrm{p}=0.03)$.

Conclusions: Higher Galectin-3 concentrations and RA adverse remodeling are related to a decreased FC in PAH patients. These findings may lead to a better characterization of PAH patients and eventually new therapeutic targets.

Keywords: Galectin-3, Pulmonary hypertension, Functional capacity. 
Introducción: La hipertensión arterial pulmonar (HAP) es una enfermedad crónica progresiva caracterizada por un remodelado en el árbol vascular pulmonar que conduce a un aumento de la resistencia vascular y disfunción ventricular derecha (VD) ${ }^{1}$. La función y remodelado ventricular derecho tiene un significativo valor pronóstico en pacientes con $\mathrm{HAP}^{2-3}$ y técnicas de deformación de imagen permiten una sensible evaluación del remodelado y la función $\mathrm{VD}^{4}$. Por otro lado, el remodelado y función auricular derecha (AD) evaluada por ecocardiografía usando speckle tracking ${ }^{5}$ y por resonancia nuclear magnética ${ }^{6}$ se asocian con eventos clínicos en pacientes con HAP.

La Galectina-3 (Gal-3) es un integrante de la familia de lectinas que contienen dominios de unión para beta-galactosidasas ${ }^{7}$. Los niveles circulantes de Gal-3 se encuentran elevados en diferentes enfermedades que cursan con fibrosis, como fibrosis hepática, renal y fibrosis pulmonar idiopática ${ }^{8}$. Por otro lado, Gal-3 se asocia a fibrosis y remodelado ventricular izquierdo en pacientes con insuficiencia cardíaca ${ }^{9}$ y pacientes con insuficiencia mitral ${ }^{10}$. En pacientes con HAP, Gal- 3 se ha asociado a marcadores ecocardiográficos de remodelado $\mathrm{VD}^{11}$.

La relación entre la Gal- 3, la función y remodelado auricular derecho (AD) y capacidad funcional (CF) en pacientes con HAP no se ha explorado. El objetivo del presente trabajo consistió en investigar la relación entre los niveles plasmáticos de Gal-3 y CF y remodelado AD en estos pacientes.

\section{Métodos}

\section{Diseño del estudio}

Estudio prospectivo observacional en que se incluyeron pacientes consecutivos con HAP, que se encontraban bajo tratamiento médico ambulatorio, entre Febrero 2014 hasta Mayo 2015. Los criterios de inclusión fueron: edad entre 18 a 60 años, diagnóstico de HAP (Clasificación de Niza Grupo 1$)^{12}$ y que estuvieran en ritmo sinusal. Se excluyeron pacientes con: embarazo, enfermedad veno-oclusiva crónica, insuficiencia cardíaca izquierda, enfermedad tromboembólica crónica, hipoxia crónica, enfermedad pulmonar crónica, enfermedad hepática crónica, fibrilación auricular u otra arritmia irregular, creatinina $>2,5 \mathrm{mg} / \mathrm{dL}$, enfermedades autoinmunes, neoplasias, estados de inflamación aguda o crónica y otras enfermedades que cursaran con fibrosis de órganos. Se recolectaron variables clínicas en todos los pacientes, incluyendo: edad, sexo, peso, talla, frecuencia cardíaca, presión arterial, fármacos al momento de la incorporación al estudio y datos del cateterismo cardíaco derecho al momento del diagnóstico. Además, en forma arbitraria, se dividieron a los pacientes en dos grupos según la distancia caminada en el test de caminata 6 minutos (> $200 \mathrm{~m}$ vs. $\leq 200 \mathrm{~m}$ ). El estudio tuvo la aprobación del Comité de Ética Institucional y los pacientes firmaron un consentimiento informado.

\section{Biomarcadores y Capacidad Funcional}

En todos los pacientes se midieron los niveles plasmáticos de Gal-3, proBNP, troponina ultrasensible y se evaluó la CF mediante test de caminata 6 min (TC6M). Además se midió PCR ultrasensible para descartar estados inflamatorios intercurrentes. La detección de Gal-3 en plasma se realizó empleando el kit comercial de BioMérieux (Marcy l'Etoile, France). El kit permite la detección de la lectina en una alícuota de $200 \mathrm{uL}$ de plasma con una sensibilidad de $3.3 \mathrm{ng} / \mathrm{mL}$.

\section{Estudios ecocardiográficos}

En todos los pacientes se realizó un ecocardiograma bidimensional con doppler al momento del ingreso al estudio. Se utilizó un equipo de ultrasonido VIVID7 (General Electric, Milwaukee, EEUU) con un transductor MS4 $(2.5 \mathrm{MHz})$. La adquisición de imágenes se realizó por un solo operador. Las mediciones fueron realizadas por dos operadores off-line, ciegos a las mediciones de biomarcadores y capacidad funcional, con el software EchoPac (versión 108.1.6 General Electric) disponible comercialmente. Se realizó un ecocardiograma estándar según la recomendación de la Sociedad Americana de Ecocardiografía, con particular cuidado en la obtención de imágenes para el análisis de las dimensiones de la aurícula derecha. El área AD se midió en una ventana apical de cuatro cámaras, en el último cuadro antes de la apertura de la válvula tricúspide. El volumen $\mathrm{AD}$ se calculó a partir del área y diámetro en ventana apical cuatro cámaras.

\section{Métodos Estadísticos}

Las variables continuas se expresaron como media \pm DS. Las variables categóricas se expresaron como números y porcentajes. Se evaluó normalidad con el test de Kolmogorov-Smirnov previa utilización de cada test. Se utilizó el Mann Whitney U Test y el método de Spearman. Se consideró como significancia estadística un valor $\mathrm{p}$ $<0,05$. Los datos se analizaron con el programa SPSS versión 15.0 (SPSS, Inc., Chicago, IL). 


\section{Resultados}

Se incluyeron 14 pacientes, la edad promedio fue $43 \pm 10$ años, el $85 \%$ mujeres. Las características clínicas y fármacos que los pacientes recibían se presentan en la Tabla 1. El $57,1 \%$ de los pacientes se encontraban en capacidad funcional II. La mayoría de los pacientes estaban en tratamiento con sildenafil $(92,8 \%)$ asociado a otro fármaco. El TC6M del grupo fue de $366 \pm 146 \mathrm{~m}$.

\begin{tabular}{l|r|}
\hline Tabla 1. Características basales y terapia. \\
\hline Característica & N $(\%)$ \\
Edad (años, promedio \pm DE) & $43 \pm 10$ \\
Sexo femenino & $12(85)$ \\
Capacidad Funcional & \\
I & $2(14,2)$ \\
II & $8(57,1)$ \\
III & $4(28,5)$ \\
IV & 0 \\
Antagonistas del receptor de endotelina & $10(71,4)$ \\
Sildenafil & $13(92,8)$ \\
Bloqueador de canales de calcio & $0(0)$ \\
Tratamiento anticoagulante oral & $11(78,5)$ \\
Oxígeno terapia & $2(14,2)$ \\
Furosemida & $8(57,1)$ \\
Espironolactona & $9(64,2)$ \\
Digoxina & $2(14,2)$ \\
&
\end{tabular}

Los resultados del cateterismo, al momento del diagnóstico fueron: presión arterial pulmonar, sistólica: 84 $\pm 9 \mathrm{mmHg}$, media: $52 \pm 6 \mathrm{mmHg}$, diastólica: $36 \pm 11$ mmHG; presión capilar pulmonar: $10 \pm 2 \mathrm{~mm} \mathrm{Hg}$; resistencia vascular pulmonar: $15 \pm 3 \mathrm{U}$ Wood.

Las variables hemodinámicas y características ecocardiográficas, al momento de la incorporación al estudio, se

Tabla 2. Características hemodinámicas y ecocardiográficas.

Característica
Frecuencia cardíaca (lpm)
Presión arterial sistólica $(\mathrm{mmHg})$
Presión arterial diastólica $(\mathrm{mmHg})$
Volumen diastólico del VD $(\mathrm{ml})$
Volumen sistólico del VD $(\mathrm{ml})$
PSAP $(\mathrm{mmHg})$
RVP (Unidades Wood)
TAPSE $(\mathrm{mm})$
Área AD 4C $(\mathrm{cm} 2)$
Volumen AD 4C (ml)
Strain global VD (\%)

Promedio \pm DS

$71 \pm 11$

$108 \pm 14$

$66 \pm 6$

$124 \pm 58$

$85 \pm 49$

$84 \pm 17$

$4,7 \pm 1,5$

$14 \pm 5$

$30 \pm 9$

$130 \pm 67$

$-12 \pm 2$
PSAP, presión sistólica de arteria pulmonar; RVP, resistencia vascular pulmonar. describen en la Tabla 2. Todos los pacientes presentaron dilatación de cavidades derechas e insuficiencia tricuspídea, entre moderada y severa.

Los niveles de los biomarcadores fueron, Gal-3: $15 \pm 3$ ng/mL; proBNP: $1.709 \pm 1.540 \mathrm{pg} / \mathrm{mL}$; Troponina ultrasensible: $13 \pm 1 \mathrm{ng} / \mathrm{L}$ y PCR ultrasensible: $16 \pm 4 \mathrm{mg} / \mathrm{dL}$. Los pacientes con menor capacidad funcional evaluada a través del TC6M, presentaron niveles mayores de Gal-3 y de proBNP. Además, presentaron mayor tamaño y menor función contráctil de la AD. La relación entre TC6M, niveles plasmáticos de Gal-3, función y remodelado $\mathrm{AD}$ se muestran en la Tabla 3. Además, se observó una correlación inversa entre el área de la $\mathrm{AD}$ y capacidad funcional medida por TC6M $(-0,71 ; \mathrm{p}=0,03)$.

Tabla 3. Remodelado y función auricular y biomarcadores según capacidad funcional.

\begin{tabular}{l|r|r|c} 
Variable & $\begin{array}{r}\text { TC6M } \\
\leq \mathbf{2 0 0 ~} \mathbf{m}\end{array}$ & $\begin{array}{c}\text { TC6M } \\
>\mathbf{2 0 0 m}\end{array}$ & P \\
\hline Galectina-3 $(\mathrm{ng} / \mathrm{mL})$ & $27,3 \pm 4,6$ & $13,7 \pm 3,8$ & 0,006 \\
Área AD 4C (cms 2) & $35,1 \pm 3,2$ & $24,5 \pm 8,5$ & 0,04 \\
Volumen AD (mL) & $151 \pm 21$ & $94 \pm 43$ & 0,04 \\
Cambio área Fraccional VD 4C (\%) & $20 \pm 10$ & $29 \pm 3$ & 0,09 \\
proBNP $(\mathrm{pg} / \mathrm{mL})$ & $6332 \pm 32$ & $45508 \pm 392$ & 0,03
\end{tabular}

\section{Discusión}

El presente estudio muestra que los niveles plasmáticos de Gal-3 y los parámetros de evaluación del remodelado $\mathrm{AD}$ se relacionan inversamente con la $\mathrm{CF}$ en pacientes con HAP.

\section{Remodelado auricular y capacidad funcional}

La función auricular izquierda se relaciona con la CF en pacientes con insuficiencia cardíaca y enfermedad coronaria ${ }^{13}$ y la función contráctil de la aurícula izquierda evaluada por strain en deportistas de alto rendimiento, aún en presencia de cavidades dilatadas, está asociada a un mayor consumo de oxígeno ${ }^{14}$. Estos trabajos están en concordancia con los datos en nuestros pacientes con HAP donde el grupo con menor CF mostró un mayor remodelado de la AD. Por otro lado se ha descrito una relación inversa entre presión sistólica de arteria pulmonar y deformación $\mathrm{AD}$ en pacientes con $\mathrm{HAP}^{15}$.

\section{Galectina-3}

Existen distintos trabajos de la relación de Gal-3 con el remodelado ventricular izquierdo evaluado por ecocar- 
diografía y fibrosis miocárdica ${ }^{16}$, incluso como marcador pronóstico en pacientes con insuficiencia cardíaca ${ }^{9}$. Por otra parte, recientemente se reportó que los niveles de Gal-3 se asocian a cambios morfológicos y funcionales del VD en pacientes con HAP ${ }^{11}$. Nuestra serie corresponde al primer reporte que relaciona un mayor remodelado de la AD con niveles de Gal-3, aunque no se demostró una correlación significativa, probablemente, por el limitado número de pacientes.

Estos hallazgos apuntan a la importancia del estudio de los mecanismos de remodelado del corazón derecho ${ }^{17}$ en estos pacientes, ya que el solo hecho de describir dilatación y disfunción puede no distinguir a muchos pacientes con pronósticos y eventualmente estrategias terapéuticas diferentes.

Este es un estudio observacional inicial que debe corro- borarse con un mayor número de pacientes. También es relevante incorporar nuevos biomarcadores y relacionarlos con otros elementos importantes en la evaluación de pacientes con HAP, como son la función endotelial y el estrés oxidativo ${ }^{18}$. Esto permitirá caracterizar y tratar de una manera más eficiente esta compleja patología. Nuevas terapias encaminadas a disminuir o antagonizar las acciones de Gal-3 podrían en el futuro constituir un nuevo blanco terapéuticos en la HAP, constituyendo un valor adicional a otros biomarcadores como BNP y troponina.

\section{Agradecimientos}

Este trabajo contó con el apoyo de los proyectos FONDAP 15130011 (a SL, PFC, HEV y LG) y FONDECYT 1141198 (a PC). 


\section{Referencias}

1. McLaughlin V, McGoon M. Pulmonary arterial hypertension. Circulation 2006; 114:1417-31.

2. Sachdev A, Villarraga HR, Frantz RP, McGoon MD, Hsiao JF, Maalouf JF et al. Right ventricular strain for prediction of survival in patients with pulmonary arterial hypertension. Chest. 2011;139:1299-309.

3. Park JH, Park MM, Farha S, Sharp J, Lundgrin E, Comhair S et al. Impaired global right ventricular longitudinal strain predicts long-term adverse outcomes in patients with pulmonary arterial hypertension. J Cardiovasc Ultrasound.2015; 23:91-9.

4. Sunbul M, Kivrak T, Durmus E, Yildizeli B, Mutlu B et al. Evaluation of right and left heart mechanics in patients with chronic thromboembolic pulmonary hypertension before and after pulmonary thromboendarterectomy. Int J Cardiovasc Imaging. 2015;31:1159-67.

5. Padeletti M, Cameli M, Lisi M, Zacà V, Tsioulpas C, Bernazzali $\mathrm{S}$ et al. Right atrial speckle tracking analysis as a novel noninvasive method for pulmonary hemodynamics assessment in patients with chronic systolic heart failure. Echocardiography. 2011;28:658-64.

6. Sato T, Tsujino I, Ohira H, Oyama-Manabe N, Ito YM, Yamada A et al. Right atrial volume and reservoir function are novel independent predictors of clinical worsening in patients with pulmonary hypertension. J Heart Lung Transplant.2015 ;34:414-23.

7. Dumic J, Dabelic S, Flogel M. Galectin-3: an open-ended story. Biochimica et Biophysica Acta 2005;1760: 616-635.

8. Mackinnon AC, Gibbons MA, Farnworth SL, Leffler H, Nilsson UJ, Delaine $\mathrm{T}$ et al. Regulation of transforming growth factor B1 driven lung fibrosis by galectin-3. Am J Respir Crit Care Med 2012;185:537-546.

9. Lok DJ, Van Der Meer P, de la Porte PW, Lipsic E, Van Wijngaarden J, Hillege HL et al. Prognostic value of Galectin-3, a novel marker of fibrosis, in patients with chronic heart failure: data from the DEAL HF study. Clin Res Cardiol 2010;99:323-8.

10. Kortekaas KA, Hoogslag GE, de Boer RA, Dokter MM, Vers- teegh MI, Braun J et al. Galectin-3 and left ventricular reverse remodeling after surgical mitral valve repair. Eur J Heart Fail 2013;15:1011-1018.

11. Fenster BE, Lasalvia L, Schroeder JD, Smyser J, Silveira LJ, Buckner JK et al. Galectin-3 levels are associated with right ventricular functional and morphologic changes in pulmonary arterial hypertension. Heart Vessels 2015. doi: 10.1007/s00380015-0691-z.

12. Simonneau G, Gatzoulis M, Adatia I, Celermajer D, Denton C, Ghofrani A et al. Updated clinical classification of pulmonary hypertension. J Am Coll Cardiol 2013;62:D34-41.

13. Kusunose K, Motoki H, Popovic ZB, Thomas JD, Klein AL, Marwick TH. Independent association of left atrial function with exercise capacity in patients with preserved ejection fraction. Heart 2012:98:1311-17.

14. Gabrielli L, Bjnens B, Brambila C, Duchateau N, Marin J, Sitges-Serra I et al. Differential atrial performance at rest and exercise in athletes: potential trigger for developing atrial dysfunction?. Scand J Med Scie Sports (in press, 2016).

15. Saha SK, Söderberg S, Lindqvist P. Association of Righ Atrial mechanics with hemodynamics and physical capacity in patients with idiopathic pulmonary arterial hypertension: insight from a single-center Cohort in Northern Sweden. Echocardiography. 2016;33:46-56.

16. de Boer RA, Voors AA, Muntendam P, van Gilst WH, van Veldhuisen DJ. Galectin-3: a novel mediator of heart failure development and progression. Eur J Heart Fail 2009;11:811-817.

17. Gabrielli L, Saavedra R, Cordova S, Llevaneras S, Sepulveda $\mathrm{P}$, Castro P. Acute changes in right ventricular synchrony and function post inhaled iloprost in pulmonary arterial hypertension patients: a myocardial deformation imaging study. Eur Heart J 2014;35 Suppl P504 (Abstract ESC 2014).

18. Gabrielli LA, Castro PF, Godoy I, Mellado R, Bourge RC, Alcaino $\mathrm{H}$ el al. Systemic oxidative stress and endothelial dysfunction is associated with an attenuated acute vascular response to inhaled prostanoid in pulmonary artery hypertension patients. J Card Fail 2011;17:1012-7. 